\title{
Signal Intensity of Lumbar Disc Herniations: Correlation With Age of Herniation for Extrusion, Protrusion, and Sequestration
}

\author{
MARKUS RAFAEL KONIECZNY, MD, ${ }^{1}$ JEREMIA REINHARDT, ${ }^{1}$ MAX PROST, MD, ${ }^{1}$ \\ CHRISTOPH SCHLEICH, MD, ${ }^{2}$ RÜDIGER KRAUSPE, MD $^{1}$ \\ ${ }^{I}$ Department of Orthopedic Surgery, University Hospital of Duesseldorf, Germany, ${ }^{2}$ Department of Radiology, University Hospital of Duesseldorf, Germany.
}

\begin{abstract}
Background: There is presently insufficient data on small groups of patients, without focus on time since herniation occurred and without establishing a valid method of measurement for signal intensity (SI) of a lumbar disc herniation (LDH) in a standard magnetic resonance imaging (MRI). SI could be reported in relation to SI of nucleus pulposus of herniated intervertebral disc, nucleus pulposus of a healthy "control" intervertebral disc, cerebral spinal fluid, or anterior anulus fibrosus. It is not known which signal intensity ratio (SIR) shows the highest correlation with time since onset of pain and how SIR of different Combined Task Forces (CTF)-types of herniation develop over time.

Methods: Out of 1053 patients, we enrolled 151 patients to a retrospective single-center analysis of standard MRIs of consecutive patients treated for LDH from February 2008 to December 2017 with confirmed (surgery, injection, or electrophysiologic testing) radicular pain by LDH and known exact date of onset of pain. We excluded patients $<18$ or $>70$ years, with chronical pain syndrome, spinal deformity, and history of prior spinal surgery on the affected spinal level. Because data did not show normal distribution, we assessed correlation by Spearman rank correlation coefficient. Correlation $(R)$ and coefficient of correlation $(C C)$ are reported.

Results: SI of LDH referenced by SI of nucleus pulposus of the affected intervertebral disc and CTF type "extrusion" showed the highest correlations with time since onset of pain $(R:-0.893$; CC: 79.7$)$, followed by CTF-type "sequestration" ( $R:-0.356 ; \mathrm{CC}: 12.7)$.

Conclusions: SIR of extrusion referenced by nucleus pulposus of the affected intervertebral disc does show a high correlation with days since onset of herniation and may be applied to monitor changes of SI of LDH after herniation in standard MRIs of the lumbar spine.

Lumbar Spine

Keywords: lumbar disc herniation, signal intensity, age of lumbar disc herniation, diagnostics, imaging, MRI, natural history, intervertebral disc, signal intensity ratio, lumbar spine, protrusion, extrusion, sequestration, radicular pain
\end{abstract}

\section{INTRODUCTION}

There is presently insufficient data on determining age of lumbar disc herniations ( $\mathrm{LDH})$, that is, time since LDH occurred, by magnetic resonance imaging (MRI). The only reports that show changes of signal intensity (SI) of LDH investigated small groups of patients without focus on time since herniation occurred and without establishing a valid method of measurement ${ }^{1,2}$ : Data on how to compare SI of a LDH of different types of MRI (different with regard to strength of magnetic field and manufacturer) is needed. A normalized ratio would enable spine specialists to compare SIs that are measured in different types of MRIs. SI of LDH could be reported in relation to SI of the nucleus pulposus of the herniated intervertebral disc, the nucleus pulposus of a healthy "control" intervertebral disc, the cerebral spinal fluid, or in relation to the anterior anulus fibrosus (Figure 1) as a signal intensity ratio (SIR). It is not known which reference is the most reliable one.

It is to be expected that change of SI differs according to Combined Task Forces (CTF)-type of herniation $^{3}$ (Figure 2) and that sequestration will show an initial increase in SI, followed by a decrease $^{2}$ (Figures 3-6).

\section{METHODS}

In a retrospective, single-center analysis, we identified consecutive patients who have been treated for $\mathrm{LDH}$ in our institution from February 2008 to December 2017. 


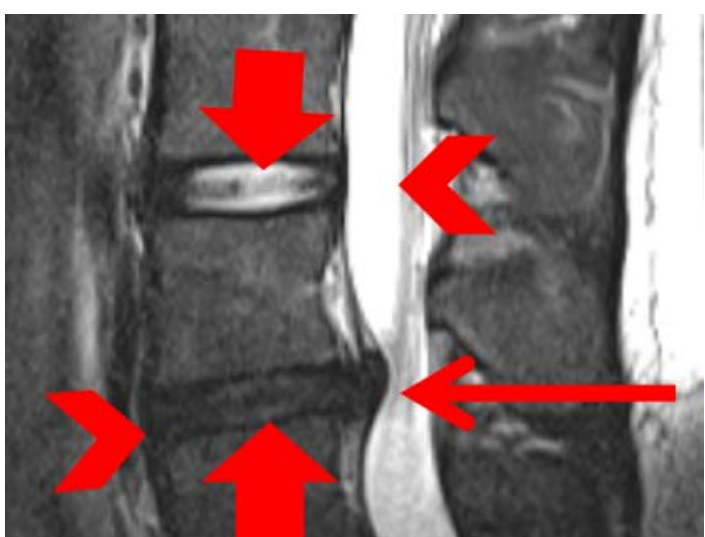

Figure 1. T2 weighted MRI of the lumbar spine, sagittal view. Nucleus pulposus of intervertebral discs L3/4 (broad downward-facing arrow) and L4/5 (broad upward facing arrow). Extrusion of L4/5 (thin arrow). Anulus fibrosus of herniated intervertebral disc (right-faced arrow head). Cerebral spinal fluid (leftfaced arrow head).

We included patients with radicular pain or neurologic deficit that matches the affected level and side in the patient's MRI, in whom LDH was verified as pain generator by surgery (reduction of pain after surgery by at least $50 \%$ ), diagnostic injection of affected nerve root (reduction of pain after injection by at least $50 \%$ ), or electrophysiologic testing that confirmed radiculopathy of the affected nerve root, and who could exactly date the onset of their radicular pain.

We excluded patients younger than 18 or older than 70 years, patients with chronical pain syndrome, spinal deformity, history of prior spinal surgery of the affected spinal level, and whose MRI was performed with less than 1.5 Tesla or with contrast agents.

We recorded demographical and clinical data of patients, location, level, and CTF-classification ${ }^{3}$ (Figure 2) of $\mathrm{LDH}$ and $\mathrm{SI}$ of $\mathrm{LDH}$, nucleus pulposus, and anulus fibrosus of the affected level and of the nucleus pulposus of a not affected intervertebral disc in the patient's MRI.

SI was measured by manually choosing a region of interest (ROI). The technique was described in former reports and showed good inter- and intraobserver reliability. ${ }^{1,4}$

SI of nucleus pulposus was determined by forming the mean of SI of anterior, median, and posterior part of each nucleus pulposus.

\section{Statistical Methods}

Statistical analysis was performed by SPSS 25 (IBM, Armonk, NY). Descriptive data are given as mean and standard error of mean. We tested all continuous variables for normal distribution by Kolmogorov-Smirnov tests. Values for SI showed no normal distribution. Thus, we assessed correlation by Spearman rank correlation coefficient. Correlation $(R)$ and coefficient of correlation (CC) are reported.

This study was approved by the local ethics committee and was conducted according to the revised Declaration of Helsinki.

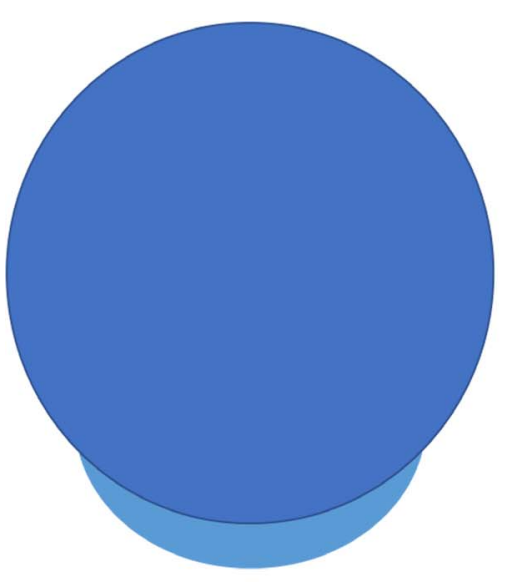

Protrusion

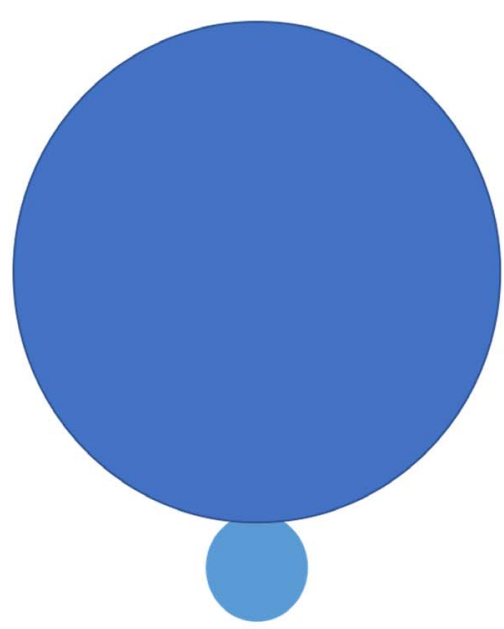

Extrusion

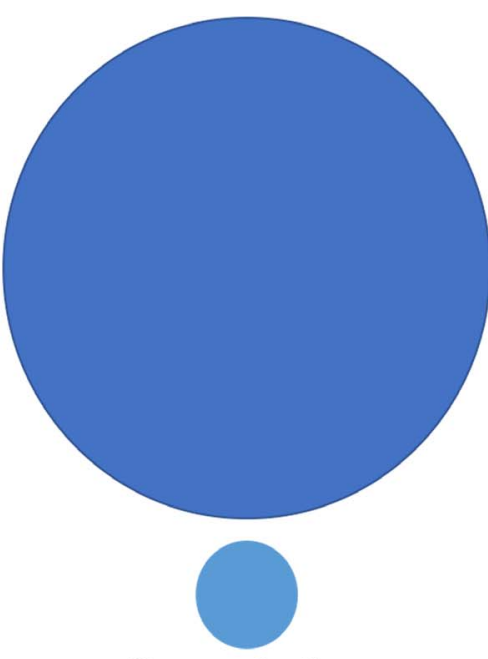

Sequestration

Figure 2. There are different types of LDH. The CTF of the North American Spine Society, American Society of Spine Radiology, and American Society of Neuroradiology established 3 different types of $\mathrm{LDH}^{3}$ : Protrusion: "A herniated disc in which the greatest plane, in any direction, between the edges of the disc material beyond the disc space is less than the distance between the edges of the base, when measured in the same plane." Extrusion: "A herniated disc in which, in at least one plane, any one distance between the edges of the disc material beyond the disc space is greater than the distance between the edges of the base in the same plane." Sequestration: "Extruded discs in which all continuity with the disc of origin is lost." 


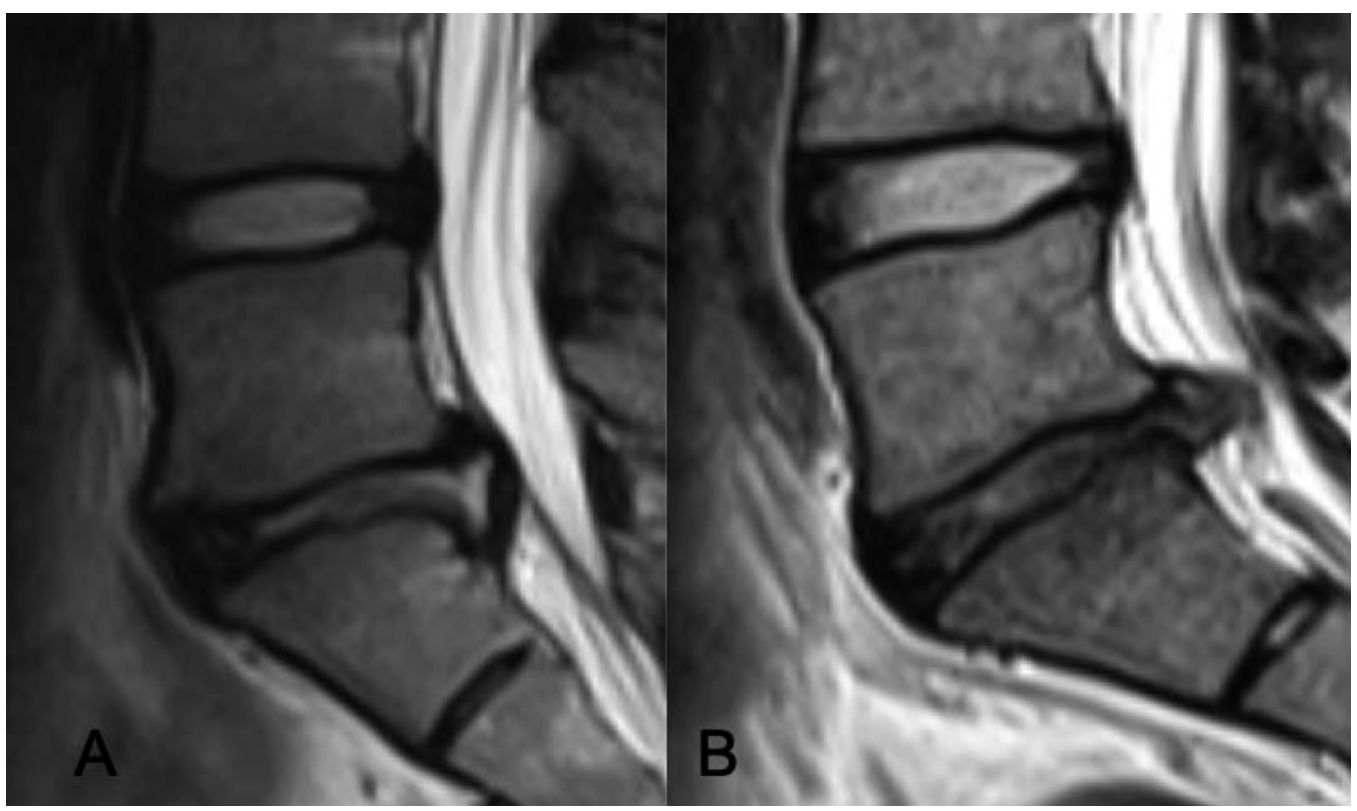

Figure 3. LDH of L5/S1 (extrusion). (A) Initial MRI 2 days after onset of pain. (B) Second MRI 4 weeks after onset of pain.

\section{RESULTS}

We identified 1053 consecutive patients by diagnostic code. After applying inclusion and exclusion criteria, we enrolled 151 patients, 64 women $(42.4 \%)$ and 87 men $(57.6 \%)$, in our analysis. Data of patients are listed in Tables 1 and 2. Data of Spearman rank correlation are given in Tables 3 and 4.

SI of herniation referenced by SI of nucleus pulposus of the affected intervertebral disc showed the highest correlations with time since onset of pain.

We found different developments of SIR of protrusions compared with extrusions and sequestrations. SIR of extrusions showed the highest correlation with time since onset of pain:

Zero to 2 weeks after onset of pain (Table 3), SIR of protrusions showed no correlation to time since

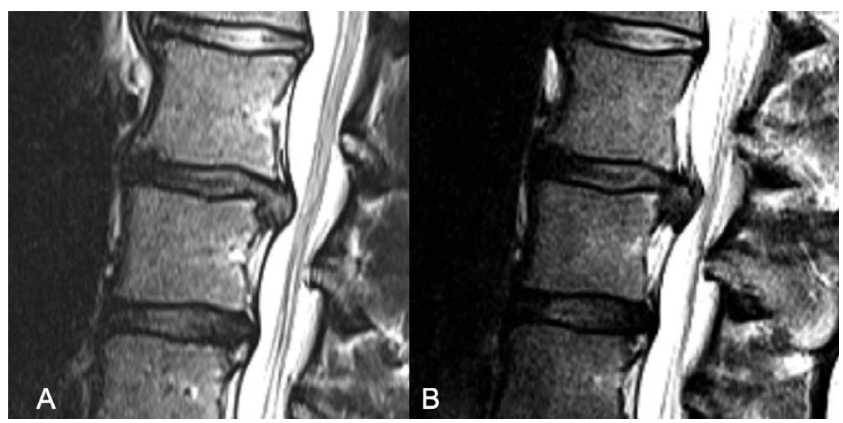

Figure 4. LDH of L2/3 (extrusion). (A) Twelve days after onset of pain. (B) Three months after onset of pain. onset of pain $(R:-0.011 ; \mathrm{CC}: 0.0)$, in contrast to sequestrations ( $R$ : -0.232 ; CC: 5.4$)$.

More than 2 weeks after onset of pain (Table 4), SIR of sequestrations showed no more correlation to time since onset of pain $(R:-0.064$; CC: 0.4$)$, and protrusions showed a low correlation $(R:-0.154$; CC: 2.4).

Extrusions showed a moderate correlation 0 to 2 weeks (Table 3$)$ after onset of pain $(R:-0.260$; CC: $6.8)$ and a high correlation with time since onset of pain more than 2 weeks (Table 4 ) after herniation $(R:-0.500$; CC: 25.0).

We performed a subgroup analysis of herniations older than 2 weeks in which we excluded all patients with a degeneration of the herniated intervertebral

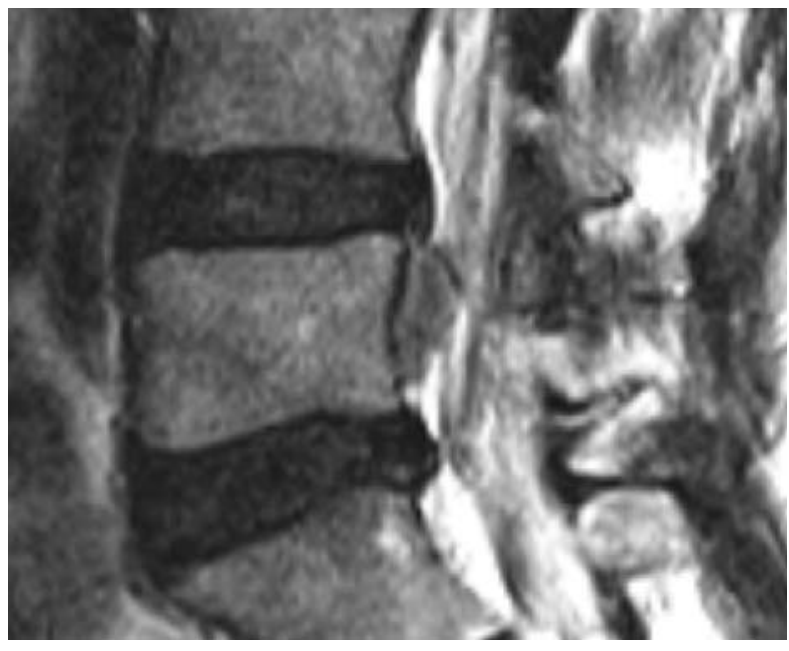

Figure 5. $L D H$ of $L 4 / 5$ (sequestration). One week after onset of pain. 


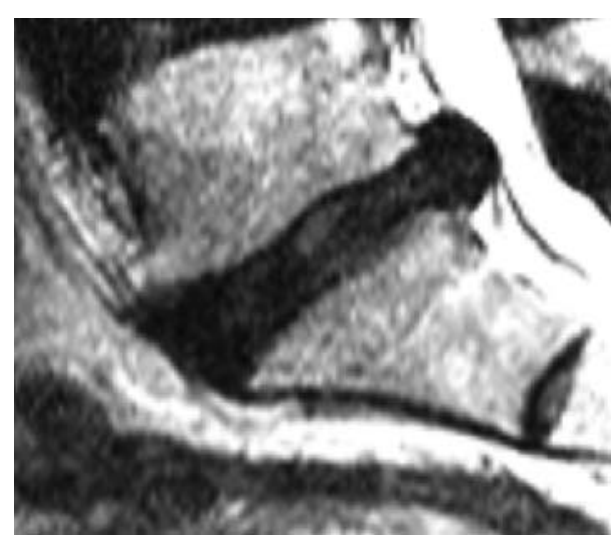

Figure 6. LDH L5/S1 (extrusion). One year after onset of pain.

disc of Pfirrmann 4 and $5 .^{5}$ We observed a substantial increase of correlation (Table 5).

SIR of extrusions showed a very high correlation $(R:-0.893$; CC: 79.7) with time since onset of pain, SIR of protrusions a low $(R:-0.209$; CC: 4.4$)$, and sequestrations showed a moderate correlation $(R$ : -0.356 ; CC: 12.7$)$ with time since onset of pain.

\section{DISCUSSION}

It is well known that many patients do present $\mathrm{LDH}$ or other degenerative changes in an MRI of their lumbar spine without any related clinical symptoms ${ }^{1,6,7}$ and that other neurogenic, musculoskeletal, immunogenic, or neoplastic diseases can mimic clinical symptoms of LDH. ${ }^{8-10}$ Thus, a clinician can never be entirely sure if an LDH in a patient's MRI with "radicular-like" pain is the source for the patient's pain, or if there is another pathology that generates the patient's pain. To verify or falsify LDH as pain generator, it would be important to gain as much information from the noninvasive MRI as possible.

Van Rijn et $\mathrm{al}^{11}$ showed that twice as many pathological findings are reported if an MRI is interpreted with clinical information - that is, "patient suffers from radiculopathy or low back pain (LBP)"- than without clinical information. Thus, if a pathology mimics radicular pain, it is not unlikely

Table 1. Level of LDH.

\begin{tabular}{lrr}
\hline & N & \% \\
\hline L1/2 & 2 & 1.3 \\
L2/3 & 4 & 2.6 \\
L3/4 & 20 & 13.2 \\
L4/5 & 65 & 43.0 \\
L5/S1 & 55 & 36.4 \\
L5/L6 & 5 & 3.3 \\
All & 151 & 100.0 \\
\hline
\end{tabular}

Table 2. CTF-Classification of LDH.

\begin{tabular}{lrr}
\hline & N & \% \\
\hline Protrusion & 18 & 11.9 \\
Extrusion & 62 & 41.1 \\
Sequestrated extrusion & 71 & 47.0 \\
All & 151 & 100.0 \\
\hline
\end{tabular}

that minor changes in a patient's MRI might be misinterpreted as pain generator, even if the pathology that is seen in the patient's MRI does not cause any clinical symptoms.

If a patient presented with onset of radicular pain less than 2 weeks before admission, and if clinicians could then verify in the patient's MRI that the according LDH herniated more than 2 weeks ago, further diagnostics would be performed that would otherwise have been delayed in search for other pathologies.

Determining age of LDH in MRIs of lumbar spines would be clinically relevant, yet there is still no data on how to perform an appropriate analysis. Establishing a reproducible method of measurement will need a distinct prospective study design. Because there is presently no data on determining age of LDH by SIR, a pilot study had to be performed to determine how SIR should be measured and to make determination of needed number of cases for further investigations possible.

Table 3. Correlation of SIR and days after onset of pain 0 to 2 weeks after onset of pain.

\begin{tabular}{|c|c|c|}
\hline Spearman-Rho & & $\begin{array}{l}\text { Days After } \\
\text { Onset of Pain }\end{array}$ \\
\hline \multirow[t]{2}{*}{ SIR protrusion/liquor } & $R$ & .063 \\
\hline & $\mathrm{N}$ & 93 \\
\hline \multirow[t]{2}{*}{ SIR extrusion/liquor } & $R$ & .028 \\
\hline & $\mathrm{N}$ & 17 \\
\hline \multirow[t]{2}{*}{ SIR sequester/liquor } & $R$ & -.216 \\
\hline & $\mathrm{N}$ & 45 \\
\hline \multirow[t]{2}{*}{ SIR protrusion/nucleus LDH } & $R$ & -.011 \\
\hline & $\mathrm{N}$ & 94 \\
\hline \multirow[t]{2}{*}{ SIR extrusion/nucleus LDH } & $R$ & -.260 \\
\hline & $\mathrm{N}$ & 17 \\
\hline \multirow[t]{2}{*}{ SIR sequester/nucleus LDH } & $R$ & -.232 \\
\hline & $\mathrm{N}$ & 46 \\
\hline \multirow[t]{2}{*}{ SIR protrusion/anterior anulus LDH } & $R$ & .074 \\
\hline & $\mathrm{N}$ & 94 \\
\hline \multirow[t]{2}{*}{ SIR extrusion/anterior anulus LDH } & $R$ & .181 \\
\hline & $\mathrm{N}$ & 17 \\
\hline \multirow[t]{2}{*}{ SIR sequester/anterior anulus LDH } & $R$ & -.112 \\
\hline & $\mathrm{N}$ & 46 \\
\hline \multirow[t]{2}{*}{ SIR protrusion/nucleus control } & $R$ & -.100 \\
\hline & $\mathrm{N}$ & 94 \\
\hline \multirow[t]{2}{*}{ SIR extrusion/nucleus control } & $R$ & .023 \\
\hline & $\mathrm{N}$ & 17 \\
\hline \multirow[t]{2}{*}{ SIR sequester/nucleus control } & $R$ & -.126 \\
\hline & $\mathrm{N}$ & 46 \\
\hline
\end{tabular}

Abbreviations: SIR, signal intensity ratio; LDH, lumbar disc herniation. 
Table 4. Spearman Correlation of SIR and days after onset of pain. More than 2 weeks after onset of pain.

\begin{tabular}{lcc}
\hline Spearman-Rho & & $\begin{array}{c}\text { Days After } \\
\text { Onset of Pain }\end{array}$ \\
\hline SIR protrusion/liquor & $R$ & .105 \\
SIR extrusion/liquor & $\mathrm{N}$ & 54 \\
SIR sequester/liquor & $R$ & .022 \\
SIR protrusion/nucleus LDH & $\mathrm{N}$ & 13 \\
SIR extrusion/nucleus LDH & $\mathrm{N}$ & 21 \\
& $R$ & -.154 \\
SIR sequester/nucleus LDH & $\mathrm{N}$ & 54 \\
SIR protrusion/anterior anulus LDH & $R$ & -.500 \\
SIR extrusion/anterior anulus LDH & $\mathrm{N}$ & 13 \\
SIR sequester/anterior anulus LDH & $R$ & -.064 \\
SIR protrusion/nucleus control & $\mathrm{N}$ & 21 \\
SIR extrusion/nucleus control & $\mathrm{N}$ & -.078 \\
SIR sequester/nucleus control & $R$ & 54 \\
& $\mathrm{~N}$ & -.286 \\
& $R$ & -.007 \\
\end{tabular}

Abbreviations: SIR, signal intensity ratio; LDH, lumbar disc herniation.

Defining an SIR in contrast to only analyzing SI is necessary to compare results of different MRIs and therefore to establish a method that may be applied by any clinician. SI of LDH may be referenced-which is needed to determine a ratio-by different values:

Nucleus pulposus of the herniated disc is the herniation's origin and should therefore be appropriate to serve as reference for changes in the herniation after "dislocation" from its "natural" environment in the intervertebral disc space.

Nucleus pulposus of a nonherniated intervertebral disc could serve as a reference for herniated disc material. However, because herniated intervertebral discs frequently show signs of degeneration, ${ }^{6,12}$ it could be a bias to use a nondegenerated intervertebral disc as reference for material that herniated from a degenerated nucleus pulposus.

Table 5. Spearman correlation of SIR and days after onset of pain. Pfirrmann 4 and 5 excluded.

\begin{tabular}{lcc}
\hline Spearman-Rho & $\mathbf{1 1 1}$ & $\begin{array}{c}\text { Days After } \\
\text { Onset of Pain }\end{array}$ \\
\hline SIR protrusion/nucleus LDH & $R$ & -.209 \\
SIR extrusion/nucleus LDH & $\mathrm{N}$ & 33 \\
SIR sequester/nucleus LDH & $R$ & -.893 \\
& $\mathrm{~N}$ & 7 \\
& $R$ & -.356 \\
\hline
\end{tabular}

Abbreviations: SIR, signal intensity ratio; LDH, lumbar disc herniation.
Cerebral spinal fluid is easy to identify in an MRI and may theoretically serve as reference for SI of other material.

Anulus fibrosus may also serve as reference. Because the posterior part of the anulus fibrosus is affected by a herniation and therefore shows changes of SI, ${ }^{12}$ we analyzed the anterior part of the anulus fibrosus in our investigation.

In our analysis we identified SIR of LDH and nucleus pulposus of the herniated disc to be the most eligible variable to monitor age of $\mathrm{LDH}$. However, LDH may lead to progressive degeneration of the affected intervertebral disc. ${ }^{12}$ If intervertebral disc degeneration progresses to Pfirrmann grade 4 or 5 , the original SI of nucleus pulposus changes significantly, which would cause a substantial bias in the measured SIR. ${ }^{5}$

This is why we performed a subgroup analysis of all cases with onset of pain more than 2 weeks after onset of pain (because the intervertebral disc could degenerate over time) and excluded patients with a grade of degeneration Pfirrmann 4 or 5 of the affected intervertebral disc. As expected, we found higher coefficients of correlation for this subgroup than in the analysis of all cases (Table 5).

LDH may be classified as protrusion, extrusion, or sequestration. ${ }^{3,13}$ These 3 different types of herniation may show different patterns of SI changes after herniation. Because protrusions are suspected to remain in the intervertebral disc space (and its chemical and biological conditions like $\mathrm{pH}$ and number of mastocytes per $\mathrm{mm}^{3}$ ), it is likely that they show minor changes compared with extrusions and sequestrations, which are exposed to the chemical and biological environment of the spinal canal. $^{3,14,15}$

As expected, protrusions, extrusions, and sequestrations showed different patterns of signal changes after onset of herniation. Protrusions showed no correlation with onset of pain 0 to 2 weeks after herniation, but a low correlation after 2 weeks. Sequestrations and extrusions correlated with time since onset of pain 0 to 2 weeks after herniation and 2 weeks or more since onset of pain, with extrusions showing the highest correlations.

A limitation of this investigation is the retrospective nature of the study. The lack of preexisting data did not allow further analyses due to underpowering: Numerous variables and 3 types of LDH had to be analyzed by 2-sided testing. However, promising results now enable clinicians and study groups to 
monitor SI of LDH with regard to time since onset of pain with the appropriate variable and knowledge of patterns of signal changes according to CTF-type of herniation.

\section{CONCLUSIONS}

SIRs of extrusion and nucleus pulposus of affected intervertebral disc do show high correlation with days since onset of herniation and may be applied to monitor changes of SI of LDH after herniation in standard MRIs of the lumbar spine. These results may be utilized to establish a staging system of the changes of SI to determine age of LDH by SIR.

\section{REFERENCES}

1. Iwabuchi M, Murakami K, Ara F, Otani K, Kikuchi S. The predictive factors for the resorption of a lumbar disc herniation on plain MRI. Fukushima J Med Sci. 2010;56(2):9197.

2. Henmi T, Sairyo K, Nakano S, et al. Natural history of extruded lumbar intervertebral disc herniation. $J$ Med Invest. 2002;49(1-2):40-43.

3. Fardon DF, Milette PC. Nomenclature and classification of lumbar disc pathology. Recommendations of the Combined Task Forces of the North American Spine Society, American Society of Spine Radiology, and American Society of Neuroradiology. Spine. 2001;26(5):E93-E113.

4. Ntilikina Y, Bahlau D, Garnon J, et al. Open versus percutaneous instrumentation in thoracolumbar fractures: magnetic resonance imaging comparison of paravertebral muscles after implant removal. J Neurosurg Spine. 2017;27(2):235-241.

5. Pfirrmann CW, Metzdorf A, Zanetti M, Hodler J, Boos N. Magnetic resonance classification of lumbar intervertebral disc degeneration. Spine (Phila Pa 1976). 2001;26(17):18731878.

6. Elfering A, Semmer N, Birkhofer D, Zanetti M, Hodler J, Boos N. Risk factors for lumbar disc degeneration: a 5-year prospective MRI study in asymptomatic individuals. Spine. 2002;27(2):125-134.

7. Boden SD, Davis DO, Dina TS, Patronas NJ, Wiesel SW. Abnormal magnetic-resonance scans of the lumbar spine in asymptomatic subjects. A prospective investigation. $J$ Bone Joint Surg Am. 1990;72(3):403-408.
8. Grimm BD, Blessinger BJ, Darden BV, Brigham CD, Kneisl JS, Laxer EB. Mimickers of lumbar radiculopathy. $J$ Am Acad Orthop Surg. 2015;23(1):7-17.

9. Park T, Lee HJ, Kim JS, Nam K. Posterior epidural disc fragment masquerading as spinal tumor: review of the literature. J Back Musculoskelet Rehabil. 2018;31(4):685-691.

10. Koda M, Mannoji C, Oikawa M, et al. Herpes zoster sciatica mimicking lumbar canal stenosis: a case report. $B M C$ Res Notes. 2015;8:320.

11. van Rijn JC, Klemetsö N, Reitsma JB, et al. Observer variation in MRI evaluation of patients suspected of lumbar disk herniation. AJR Am J Roentgenol. 2005;184(1):299-303.

12. Lama P, Le Maitre CL, Dolan P, Tarlton JF, Harding IJ, Adams MA. Do intervertebral discs degenerate before they herniate, or after? Bone Joint J. 2013;95-B(8):1127-1133.

13. Oh KJ, Lee JW, Yun BL, et al. Comparison of MR imaging findings between extraligamentous and subligamentous disk herniations in the lumbar spine. AJNR Am J Neuroradiol. 2013;34(3):683-687.

14. Di Martino A, Merlini L, Faldini C. Autoimmunity in intervertebral disc herniation: from bench to bedside. Expert Opin Ther Targets. 2013;17(12):1461-1470.

15. de Souza Grava AL, Ferrari LF, Defino HL. Cytokine inhibition and time-related influence of inflammatory stimuli on the hyperalgesia induced by the nucleus pulposus. Eur Spine $J$. 2012;21(3):537-545.

Disclosures and COI: The authors received no funding for this study and report no conflicts of interest. The study was approved by the local ethics committee (Registration Number 5503).

Corresponding Author: Markus Rafael Konieczny, MD, Department of Orthopedic Surgery, University Hospital of Duesseldorf, Germany, Moorenstr. 5, 40225 Duesseldorf, Germany. Phone: +49 (0)211 81 18314; Email: markus.konieczny@ med.uni-duesseldorf.de.

Published 29 February 2020

This manuscript is generously published free of charge by ISASS, the International Society for the Advancement of Spine Surgery. Copyright (C) 2020 ISASS. To see more or order reprints or permissions, see http://ijssurgery.com. 